\title{
Corrigendum: Topological excitations in a kagome magnet
}

\author{
Manuel Pereiro, Dmitry Yudin, Jonathan Chico, Corina Etz, Olle Eriksson \& Anders Bergman
}

Nature Communications 5:4815 doi: 10.1038/ncomms5815 (2014); Published 8 Sep 2014; Updated 20 Jul 2015

This Article contains an unintentional error for the excitation energies provided by the external magnetic field. The first excitation was carried out with an energy of $26.1 \mathrm{meV}$ and the second excitation with an energy of $81.4 \mathrm{meV}$, instead of 261 and $814 \mathrm{meV}$, respectively. This error affects the $y$ axis of Fig. 2a and Supplementary Fig. 6. In addition, the edge and bulk magnon states in Fig. 2b,c were presented for these wrong energies, instead of the correct energies used for the excitations. Corrected versions of Fig. 2 and Supplementary Fig. 6 appear below. These errors throughout the Article do not affect the content and conclusions of the paper.

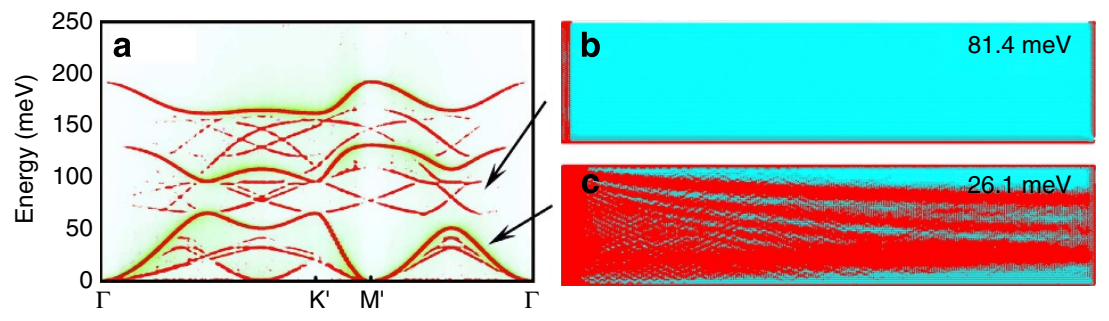

Figure 2

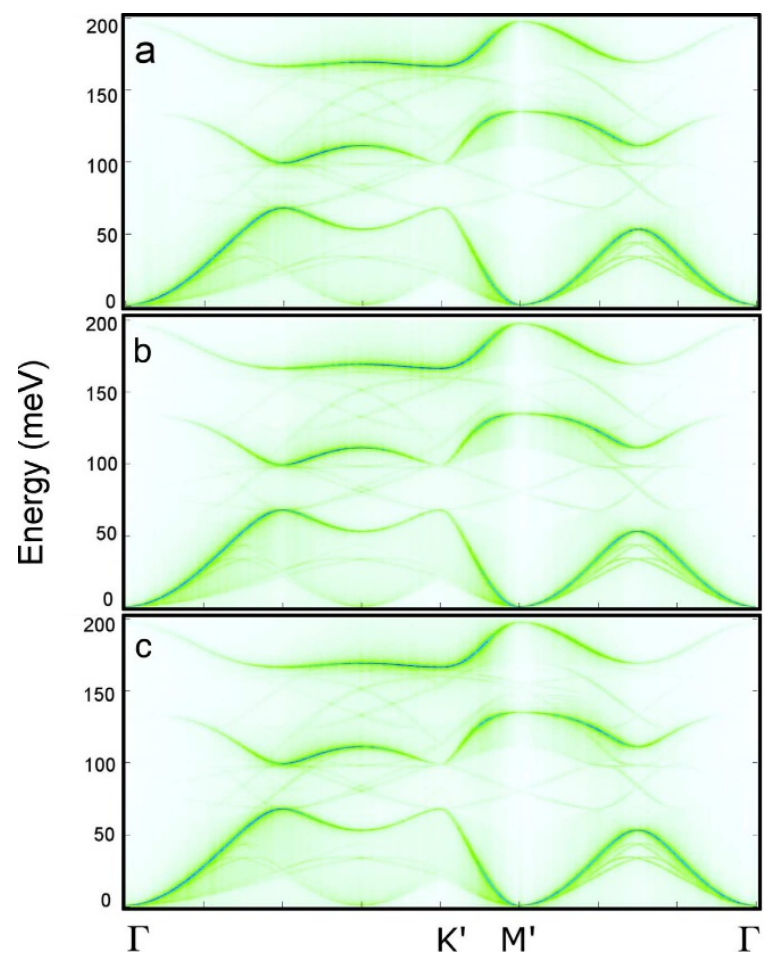

